Acta Cryst. (1954). 7, 559

\title{
The Crystal Structure of Formamide*
}

\author{
By Joshua Ladell and Benjamin Post \\ Polytechnic Institute of Brooklyn, Brooklyn 2, New York, U.S.A.
}

(Received 17 February 1954 and in revised form 9 March 1954)

\begin{abstract}
Formamide, $\mathrm{HCONH}_{2}$, melts at $2.5^{\circ} \mathrm{C}$. On the basis of an X-ray diffraction investigation conducted at $-50^{\circ} \mathrm{C}$., it was found that the compound crystallizes in a monoclinic unit cell with: $a=3 \cdot 69 \pm 0 \cdot 01, b=9 \cdot 18 \pm 0 \cdot 025, c=6 \cdot 87 \pm 0 \cdot 02 \AA, \beta=98 \pm \frac{10}{4}$. The space group is $P 2_{1} / n$. There are four molecules per unit cell.

The configuration and orientation of the molecule have been determined, using complete (three-dimensional) X-ray diffraction data. The structure consists of puckered sheets of formamide molecules; adjacent sheets are separated by about $3 \cdot 1 \AA$. Within the sheets pairs of formamide molecules associate about centers of symmetry to form almost coplanar 'bimolecular' units. Puckering of the sheets results from the tilt of these bimolecular units relative to one another.

Sheets of formamide molecules are approximately parallel to (101). Within each sheet hydrogen bonds of two types exist: one type, $2 \cdot \mathbf{9 3}_{5} \AA$ long, links monomers together to form 'bimolecular' units; the other type, $\mathbf{2 \cdot 8 8 _ { 0 }} \AA$ long, links bimolecular units together to form sheets.

The structure may be alternatively described as consisting of chains of formamide molecules cross-linked by hydrogen bonds to form sheets.

Bond lengths within the molecule are $\mathrm{C}-\mathrm{O}=1 \cdot 25_{5}, \mathrm{C}-\mathrm{N}=1 \cdot 30_{0} \AA$. The bond angle $\mathrm{O}-\mathrm{C}-\mathrm{N}$ is $121 \cdot 5^{\circ}$. The probable error in bond lengths is $0.013 \AA$.
\end{abstract}

\section{Introduction}

Many careful studies of the amide group<smiles>[R2]NC([R4])[R4]</smiles>

in amino acids and related compounds have been carried out in the past few years. As a result, the configuration of this group in a wide variety of organic molecules is now well known. Less is known of the structure of the terminal amide group in organic molecules, although a number of interesting problems are associated with the crystal structures of such molecules, e.g. the extent of utilization of the two available hydrogen atoms for hydrogen bonding, the relative length of these bonds, the relative lengths of the $\mathrm{C}-\mathrm{O}$ and $\mathrm{C}-\mathrm{N}$ bonds, the extent of coplanarity of the three bonds emanating from the nitrogen atom, etc.

When this investigation was begun, the structure of only one molecule containing this (terminal amide) group had been determined (acetamide (Senti \& Harker, 1940)). Within the past year additional determinations of structures having terminal amide groups have been made; these are discussed below.

Formamide $\left(\mathrm{HCONH}_{2}\right)$ was selected for this investigation primarily because the terminal amide group

* This work was supported by the Office of Naval Research. Taken from a dissertation submitted by $J$. Ladell in partial fulfilment of the requirements for the Ph.D. is virtually the entire molecule; the interpretation of the structure is not complicated by the presence of large substituent groups in the molecule.

\section{Experimental}

Thin-walled glass capillary tubes were filled with carefully purified formamide (m.p. $2 \cdot 5^{\circ}$ C.) and sealed. Single crystals were grown in the capillary tubes and maintained at $-50 \pm 5^{\circ} \mathrm{C}$. using previously described techniques (Post, Schwartz \& Fankuchen, 1951). Optical examination of the crystals, using polarized light, gave no indication of solid-phase transitions in the temperature range $-60^{\circ} \mathrm{C}$. to the melting point.

The crystals almost invariably grew along what was later identified as the $a$ axis of the monoclinic unit cell. Since alternative orientations of the crystals could not readily be obtained, it was found convenient to use both Weissenberg and precession cameras to accumulate full three-dimensional data.

A Weissenberg camera was built (Steinfink, Ladell, Post \& Fankuchen, 1953) which made possible the convenient investigation, at low temperatures, of the full equi-angular range attainable with the normal instrument. Zero- and upper-layer equi-inclination diagrams were obtained using inclination angles up to $35^{\circ}$. In this way $h k l$ data, with $h=0-3$, were recorded. Other regions of reciprocal space were explored with the precession camera.

$\mathrm{Cu} K$ radiation and multiple-film techniques were used for Weissenberg diagrams; Mo $K$ radiation was used for precession diagrams; each precession diagram was recorded several times for varying time intervals 
to make certain all reflections could be correlated with a linear scale. Intensities were estimated visually by comparison with standard intensity scales. Absorption corrections were unnecessary, since in no case was the crystal size greater than a small fraction of the optimum crystal diameter. The usual Lorentz and polarization corrections were applied and all intensities were put on the same scale.

A total of 324 independent reflections was recorded.

Measurements of precession diagrams and diffractometer traces of polycrystalline formamide indicated that the crystals were monoclinic. The diagrams were indexed on the basis of a unit cell with dimensions:

$$
\begin{gathered}
a=3 \cdot 69 \pm 0 \cdot 01, b=9 \cdot 18 \pm 0 \cdot 025, c=6 \cdot 87 \pm 0 \cdot 02 \AA, \\
\beta=98 \pm 1^{\circ} .
\end{gathered}
$$

Reflections of the type $0 k 0$ with $k$ odd, $h 00$ with $h$ odd and $h 0 l$ with $h+l$ odd were systematically absent from all single-crystal diagrams; the space group is therefore $P 2_{1} / n$ (which is equivalent to $P 2_{1} / c$ upon the appropriate axial transformations).

The density of liquid formamide at $20^{\circ} \mathrm{C}$. is $1 \cdot 13$ g.cm. ${ }^{-3}$; if it is assumed that there are four molecules per unit cell, the calculated (X-ray) density is 1.26 g.cm. ${ }^{-3}$ (at $-50^{\circ}$ C.). This is in good agreement with the value listed for liquid formamide at room temperature if the difference in temperature and contraction of the liquid on freezing are taken into account. The asymmetric unit is therefore one molecule of formamide.

\section{Determination of the structure}

Patterson $(0 k l)$ and $(h k 0)$ projections were computed. The $(h k 0)$ projection could not be successfully interpreted. The $(0 \mathrm{kl})$ projection was more useful; one large peak appeared clearly to be due to an intra-molecular nitrogen-oxygen interaction; other peaks in this projection indicated that at least one, and possibly two, atoms were situated near a screw axis.

It was expected that the $(0 k l)$ electron-density projection (i.e. down the short $a$ axis) would show satisfactory resolution of the atoms. Several molecular arrangements consistent with the Patterson (0kl) projection were tried. After several false starts, an $(0 k l)$ electron-density map was computed which showed satisfactory agreement with the assumed model. The projection was refined by the usual Fourier iteration process; the final map showed the molecule clearly resolved.

Efforts were then made to determine the $x$ parameters of the atoms. A Patterson-Harker section at $u, \frac{1}{2}, w$ was computed. The approximate $x$ parameter of one atom (it later proved to be the nitrogen atom) could readily be deduced from this section. The $x$ parameters of the other atoms could not be determined with any precision because of superposition of the carbon-carbon and oxygen-oxygen interactions. The position of the unresolved peak did indicate, however, that the carbon and oxygen atoms were near $x=\frac{1}{2}$.
An $(h k 0)$ electron-density map was then computed on the basis of approximate $x$ parameters determined from the Harker synthesis, and $y$ parameters known from the $(0 k l)$ projection; the map was refined in the usual way and the result is shown in Fig. 1.

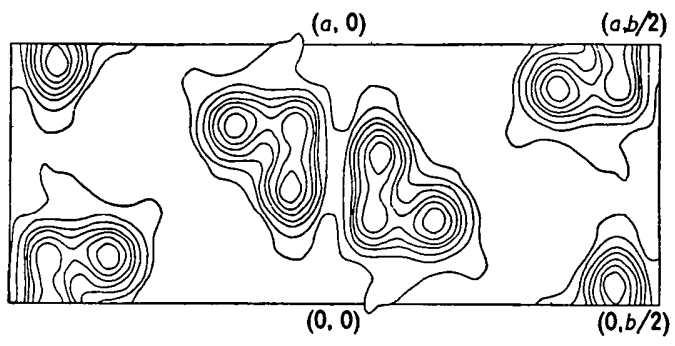

Fig. 1. The (hk0) electron-density projection. Contours are drawn at arbitrary intervals (approximately 1 e. $\AA^{-2}$ ).

The projections appeared to be satisfactory in all but one respect; bond lengths calculated from the two projections were considerably shorter than expected: the carbon-oxygen bond was $1 \cdot 19 \AA$, and the carbonnitrogen bond only $1.20 \AA$.

It was felt that the anomalous bond lengths might reflect errors in the $x$ parameter of the carbon atom resulting from the small number of orders of $h$ used in the $(h k 0)$ projection and from overlap of carbon with nitrogen and oxygen peaks; the overlap is clearly visible in the $(h k 0)$ electron-density map (Fig. 1). The $x$ parameter of the carbon atom was varied systematically in the vicinity of the position indicated by the Fourier map, but the discrepancy factor $R$ was a minimum (18\%) for the carbon position indicated by the $(h k 0)$ map. An $(h k 0)$ difference synthesis also confirmed this carbon position.

At this point, contributions of the hydrogen atoms to the structure factors were considered; inclusion of hydrogen atoms in the calculations resulted in several sign changes for weak reflections; inclusion of these changes in the summation improved considerably the appearance of the (0kl) Fourier map (Fig. 2). Bond

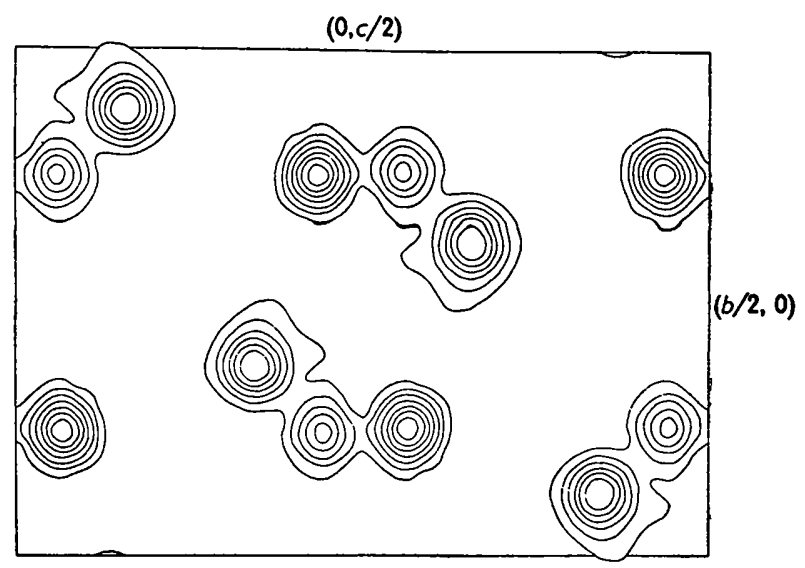

Fig. 2. Final $(0 \mathrm{kl})$ electron-density projection. Contours are drawn at arbitrary intervals (approximately 1 e. $\AA^{-2}$ ). 


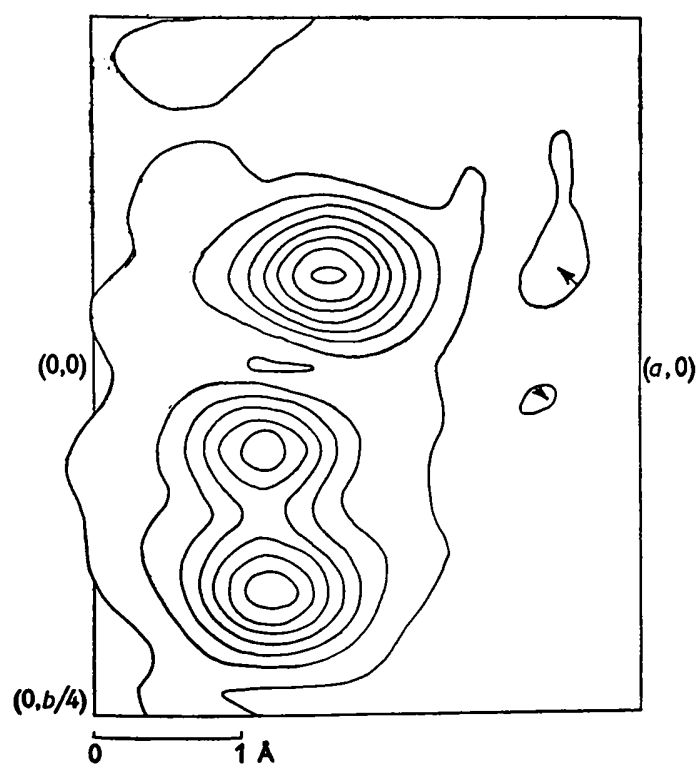

Fig. 3. Half-cell (bounded) projection down $c$ axis. One asymmetric unit is shown. Contours are drawn at arbitrary intervals (approximately 1 e. $\AA^{-2}$ ). Negative regions are indicated by arrows.

lengths computed from this corrected projection and from the $(h k 0)$ projection were: $\mathrm{C}-\mathrm{O}=1 \cdot 24 \AA \mathrm{A}, \mathrm{C}-\mathrm{N}=$ $1.27 \AA$.

Parameters indicated by the final $(0 k l)$ and $(h k 0)$ projections are listed in Table 1. Back-shift corrections were computed for both sets of parameters. Since the
$(0 \mathrm{kl})$ projection is based in part on electron density not directly observable on the map (hydrogen atoms), the final 'back-shift parameters' were further refined by the method of least squares. In this calculation, off-diagonal terms were neglected. These results are also listed in Table 1.

Further refinement of atomic parameters, using bounded projections (Booth, 1948), was then carried out. The contents of the unit cell between $z=0$ and $z=\frac{1}{2}$ were projected on to the basal plane. In this electron-density projection all observed $F$ 's were included except those for which $l$ was even and not equal to zero. (These cancel out in the summation; 193 terms remained.) Signs for observed structure factors were calculated using parameters listed in Table 1. The projection was refined in the usual way. One asymmetric unit of the resulting electron density map is shown in Fig. 3.

As can be seen in Fig. 3, the carbon, nitrogen and oxygen atoms of one molecule all lie in the region between $x=\frac{1}{4}$ and $x=\frac{1}{2}$. A bounded projection down the $a$ axis, projecting the contents of the unit cell between $x=\frac{1}{4}$ and $x=\frac{1}{2}$, was therefore computed. In this projection all $F$ 's are included in the summation except those for which $h \neq 0, h \equiv 0 \bmod 4$. A half-cell projection down the $a$ axis would have required the omission from the summation of all even orders of $h$ except $h=0 ; 322$ terms were included in the quarter-cell projection compared with 225 terms which would have been used in a half-cell projection. Fig. 4 lists a sequence of maps showing the contents

Table 1. Parameters determined from two-dimensional projections

$(0 k l)$

Fourier

Back-shift corrections Implied

Least squares

$(h k 0)$

Fourier

Back-shift corrections

Implied

\begin{tabular}{|c|c|c|}
\hline \multicolumn{3}{|c|}{ Oxygen } \\
\hline$x$ & $y$ & $z$ \\
\hline - & -0.066 & $0 \cdot 245$ \\
\hline - & -0.002 & 0.000 \\
\hline 一 & -0.068 & 0.245 \\
\hline - & -0.069 & 0.246 \\
\hline
\end{tabular}

\begin{tabular}{ccr}
\multicolumn{3}{c}{ Nitrogen } \\
$x$ & $y$ & $z$ \\
- & 0.157 & 0.126 \\
- & 0.003 & -0.002 \\
- & 0.160 & 0.124 \\
- & 0.159 & 0.128
\end{tabular}

\begin{tabular}{ccc} 
Carbon \\
\hline$x$ & $y$ & $z$ \\
- & 0.058 & 0.256 \\
- & 0.002 & 0.004 \\
- & 0.060 & 0.260 \\
- & 0.061 & 0.257
\end{tabular}

\begin{tabular}{|c|c|c|c|c|c|c|c|c|}
\hline 0.431 & -0.068 & 一 & 0.322 & 0.161 & - & $0 \cdot 330$ & 0.061 & - \\
\hline-0.006 & -0.001 & - & 0.003 & 0.000 & - & -0.012 & 0.000 & - \\
\hline 0.42 & -0.069 & - & 0.325 & $0 \cdot 161$ & - & $0.318 *$ & 0.061 & \\
\hline
\end{tabular}

* Not considered reliable because of partial superposition of oxygen peak.

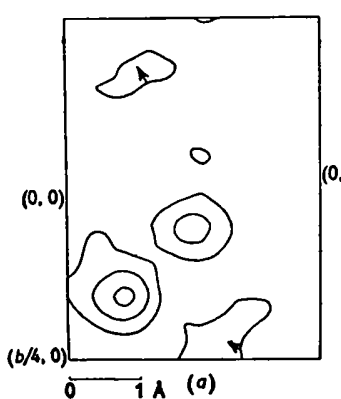

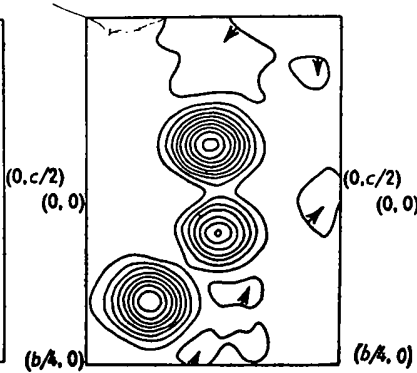

(b)

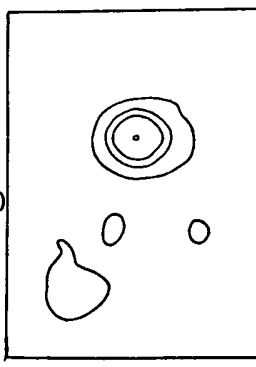

(c)

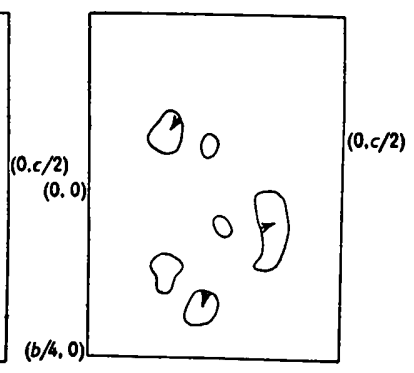

(d)

Fig. 4. One-quarter cell projection down $a$ axis. One asymmetric unit is shown in the four accompanying diagrams (see text). Contours are at arbitrary intervals (approximately $\frac{1}{2}$ e. $\left.\AA^{-2}\right)$. Negative regions are indicated by arrows. 
Table 2. Parameters determined from bounded projections

\begin{tabular}{|c|c|c|c|c|c|c|c|c|c|}
\hline & \multicolumn{3}{|c|}{ Oxygen } & \multicolumn{3}{|c|}{ Nitrogen } & \multicolumn{3}{|c|}{ Carbon } \\
\hline & $x$ & $y$ & $\boldsymbol{z}$ & $x$ & $y$ & $z$ & $x$ & $y$ & $z$ \\
\hline $\begin{array}{l}\text { Half cell down } c \\
\text { Back-shift corrections } \\
\text { Implied }\end{array}$ & $\begin{array}{l}0 \cdot 4296 \\
0 \cdot 004 \\
0 \cdot 4336\end{array}$ & $\begin{array}{c}-0.0660 \\
0 \cdot 000 \\
-0.0660\end{array}$ & E & $\begin{array}{l}0.3219 \\
0 \cdot 008 \\
0.3299\end{array}$ & $\begin{array}{c}0.1605 \\
-0 \cdot 001 \\
0 \cdot 1595\end{array}$ & - & $\begin{array}{l}0 \cdot 3078 \\
0 \cdot 002 \\
0 \cdot 3098\end{array}$ & $\begin{array}{c}0.0601 \\
-0.001 \\
0.0591\end{array}$ & $\bar{z}$ \\
\hline $\begin{array}{l}\text { Quarter cell down } a \\
\text { Back-shift corrections } \\
\text { Implied } \\
\text { Final }\end{array}$ & $\frac{\bar{Z}}{\overline{0.434}}$ & $\begin{array}{l}-0.0672 \\
-0.001 \\
-0.0682 \\
-0.067\end{array}$ & $\begin{array}{l}0 \cdot 2435 \\
0 \cdot 000 \\
0 \cdot 2435 \\
0 \cdot 244\end{array}$ & $\frac{\bar{Z}}{\overline{0}}$ & $\begin{array}{l}0 \cdot 1573 \\
0 \cdot 0006 \\
0 \cdot 1579 \\
0 \cdot 159\end{array}$ & $\begin{array}{l}0 \cdot 1282 \\
0 \cdot 000 \\
0 \cdot 1282 \\
0 \cdot 128\end{array}$ & $\bar{z}$ & $\begin{array}{l}0.0590 \\
0 \cdot 000 \\
0 \cdot 0590 \\
0 \cdot 059\end{array}$ & $\begin{array}{l}0.2601 \\
0 \cdot 001 \\
0 \cdot 2611 \\
0.261\end{array}$ \\
\hline \multicolumn{10}{|c|}{ Hydrogen atoms were assumed at: } \\
\hline & 0.231 & 0.262 & $0 \cdot 176$ & 0.420 & $0 \cdot 124$ & -0.010 & $0 \cdot 184$ & 0.085 & $0 \cdot 389$ \\
\hline
\end{tabular}

of the unit cell between $x=0$ and $x=1$ (Fig. 4(a)), $x=\frac{1}{4}$ to $x=\frac{1}{2}$ (Fig. 4(b)), $x=\frac{1}{2}$ to $x=\frac{3}{4}$ (Fig. 4(c)), and $x=\frac{3}{4}$ to $x=1$ (Fig. $4(d)$ ). These maps are in turn generated from the expression

$$
\varrho_{\frac{1}{t}}^{\frac{1}{2}}(y, z)=\int_{\frac{1}{z}}^{\frac{1}{z}} \varrho(x, y, z) d x
$$

when the symmetry elements of the unit cell are applied. Atomic locations were determined from Fig. $4(b)$; contributions to the atoms as shown in Fig. $4(a),(c)$ and $(d)$ were not considered in the determination of atomic parameters since they represent fringes of atoms in which spherical symmetry no longer (necessarily) exists.

Back-shift corrections were computed for both bounded projections. These and the implied final parameters are listed in Table 2.

Observed and calculated values of $F$ are listed in Table 3.* Temperature corrections were included in the above values of $F_{c}$ using

$$
\boldsymbol{F}_{c}=\overline{\boldsymbol{F}}_{h k l} \exp \left[-\left(\alpha_{1} h+\alpha_{2} k+\alpha_{3} l+\alpha_{4} h l\right)\right],
$$

where $\bar{F}_{h k l}$ is the geometric structure factor. Values of $\alpha_{1}, \alpha_{2}, \alpha_{3}$, and $\alpha_{4}$ were computed by least-square methods by minimizing the function

$$
\sum w_{h k l}\left\{\ln \left[\frac{F_{o}}{\bar{F}_{h k l} \cdot \exp \left[-\alpha_{0}-\alpha_{1} h^{2}-\alpha_{2} k^{2}-\alpha_{3} l^{2}-\alpha_{4} h l\right]}\right]\right\}^{2},
$$

$\exp \left[-\alpha_{0}\right]$ is a scaling factor and $w_{h k l}$ is a 'weighting' function. $w_{h k l}=\left|F_{o}\right|$ was used, favoring the medium and strong reflections. Values of the temperature parameters were found to be: $\alpha_{0}=-1.34, \alpha_{1}=0.13$, $\alpha_{2}=0.008, \alpha_{3}=0.020, \alpha_{4}=0.030$.

Contributions due to hydrogen atoms were included

* Table 3, comprising 340 observed and calculated $F$ values, has been withdrawn and is deposited as Document No. 4230 with the ADI Auxiliary Publications Project, Photoduplication Service, Library of Congress, Washington 25, D.C., U.S.A. A copy may be secured by citing the Document number and by remitting $\$ 1.25$ for photoprints, or $\$ 1.25$ for $35 \mathrm{~mm}$. microfilm. Advance payment is required. Make checks or money orders payable to: Chief, Photoduplication Service, Library of Congress. Copies may also be obtained from the author. in the calculation of structure. factors. It was assumed that hydrogen atoms were at $1 \cdot 1 \AA$ from the nitrogen atom in the direction of adjacent oxygen atoms. A hydrogen atom was also assumed in the plane of $\mathrm{N}-\mathrm{C}-0,1 \cdot 1 \AA$ away from the carbon atom such that the angle $\mathrm{H}-\mathrm{C}-\mathrm{N}$ was $120^{\circ}$. Atomic positions assumed for the hydrogen atoms are listed in Table 2.

\section{Estimation of accuracy}

The magnitude of random errors in atomic coordinates was investigated using Cruickshank's expressions (Cruickshank, 1949):

$$
\begin{aligned}
\sigma\left(\frac{\partial \varrho}{\partial x_{j}}\right) & =\frac{2 \pi}{a_{j} V}\left[\sum_{h_{1}} \sum_{h_{2}} \sum_{h_{3}} h_{j}^{2} \Delta F^{2}\right]^{\frac{z}{z}}, \\
\sigma(x) & =\left[\frac{\partial^{2} \varrho}{\partial x^{2}} \sin ^{2} \beta\right]^{-1}\left\{\sigma^{2}\left(\frac{\partial \varrho}{\partial x}\right)-\sigma^{2}\left(\frac{\partial \varrho}{\partial z}\right) \cos ^{2} \beta\right\}^{\frac{1}{z}}, \\
\sigma(z) & =\left[\frac{\partial^{2} \varrho}{\partial z^{2}} \sin ^{2} \beta\right]^{-1}\left\{\sigma^{2}\left(\frac{\partial \varrho}{\partial z}\right)-\sigma^{2}\left(\frac{\partial \varrho}{\partial x}\right) \cos ^{2} \beta\right\}^{\frac{1}{z}}, \\
\sigma(y) & =\left[\frac{\partial^{2} \varrho}{\partial y^{2}}\right]^{-1} \sigma\left(\frac{\partial \varrho}{\partial y}\right) .
\end{aligned}
$$

The values of $\partial^{2} \varrho / \partial x^{2}$ etc. were determined from the values of the electron density in the neighborhood of the final Fourier peaks. Mean values for all the atoms are:

$$
\partial^{2} \varrho / \partial x^{2}=41, \partial^{2} \varrho / \partial y^{2}=82, \partial^{2} \varrho / \partial z^{2}=66 \text { e. } \AA^{-5} \text {. }
$$

The standard deviations of the partial derivatives of $\varrho$ were found to be:

$$
\begin{gathered}
\sigma(\partial \varrho / \partial x)=0.840, \sigma(\partial \varrho / \partial y)=0.852, \\
\sigma(\partial \varrho / \partial z)=0.878 \mathrm{e} . \AA^{-4}
\end{gathered}
$$

The standard deviations of atomic positions are:

$$
\sigma(x)=0.021, \sigma(y)=0.010, \sigma(z)=0.013 \AA .
$$

The standard deviations in bond length were calculated by compounding the above values in the bond directions and multiplying by $/ 2$. They are:

$0.018 \AA$ for $\mathrm{C}-\mathrm{O}, 0.017 \AA$ for $\mathrm{C}-\mathrm{N}$, $0.017 \AA$ for $\mathrm{N}_{1}-\mathrm{H} \cdots \mathrm{O}_{3}$, and $0.019 \AA$ for $\mathrm{N}_{1}-\mathrm{O}_{2}$. 
The corresponding probable errors in bond lengths are less than $0.013 \AA$.

The discrepancy factors for the final coordinates are:

$$
\begin{gathered}
R_{h k l}=19 \cdot 0 \%, R_{h k 0}=17 \cdot 3 \%, R_{h 0 l}=26.0 \%, \\
\text { and } R_{0 k l}=13.5 \% .
\end{gathered}
$$

In this determination only observed structure factors were considered. The reflections (002), (101), (10) $)$, (110), and (21 $\overline{1})$ were considered unreliable because of extinction effects and were therefore omitted in the determination of the discrepancy factors. (Calculated structure factors were used for these reflections in the final Fourier projections.)

\section{Molecular environment}

The molecular arrangement in the crystal may be best visualized as follows: Individual formamide molecules associate to form dimers; * the dimers are held together by two $\mathrm{N}-\mathrm{H} \cdots \mathrm{O}$ bonds. Each dimer, in turn, is bonded by hydrogen bonds of the same type, to four other dimeric units. The structure is made up of

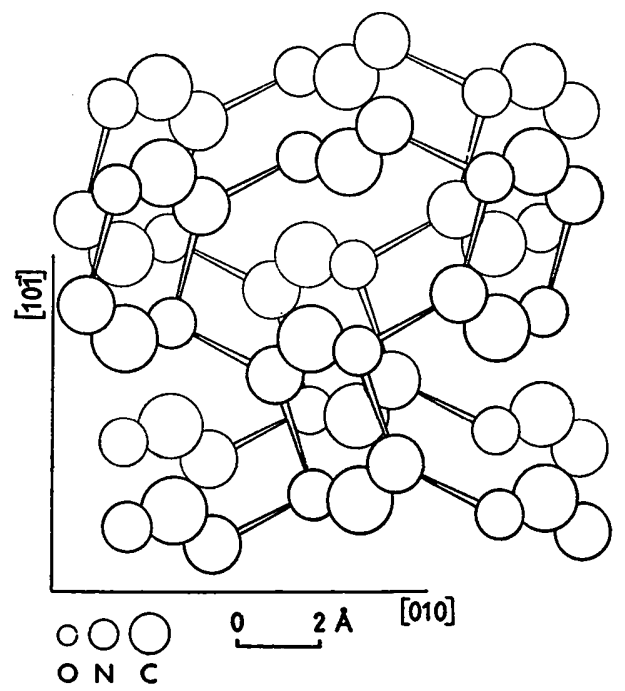

Fig. 5. Molecular arrangement in formamide crystal. View is normal to the (101) plane. Molecules indicated by thin lines are under those in heavy lines. Double lines indicate hydrogen bonds.

puckered sheets of dimeric units held together, within the sheets, by strong $\mathrm{N}-\mathrm{H} \cdots \mathrm{O}$ bonds. The arrangement of molecules in the crystal is shown in Fig. 5. All six heavy atoms within one dimeric unit are almost coplanar. The degree of planarity may be described

* The referee has correctly pointed out that since the hydrogen bonds between 'dimeric' units are apparently stronger than those making up the 'dimers', it would be more accurate to speak only of sheets of formamide molecules without specific reference to 'dimeric' units. We have retained the term 'dimer', however, using it in a restricted sense in this discussion to emphasize a geometrical aspect of the molecular sheets, i.e. the tendency of pairs of molecules to associate about a center of symmetry in almost coplanar units. as follows: Because of the presence of a center of symmetry at the center of the dimer, the two nitrogen and two oxygen atoms within any one dimer are necessarily coplanar; the two carbon atoms are only $0.094 \AA$ out of this plane. The best plane through all six (heavy) atoms in the dimeric unit is no more than $0.045 \AA$ from any atom in the group. Each dimeric 'plane' is tilted at an angle of $19^{\circ}$ to the (101) plane; the plane of centers of symmetry (i.e. the centers of dimers) within any one sheet is parallel to (101).

Adjacent sheets are held together by van der Waals forces. The average distance between sheets is 3.1 $\AA$. The closest approach between atoms in different sheets is $3.39 \AA$, i.e. between a carbon atom of one layer and a nitrogen atom of another.

Successive sheets of dimers are arranged so as to bring the bulk of one dimeric unit above the largest holes of the sheet below; this displacement is parallel to (101) and accounts for the monoclinic angle.

\section{Molecular dimensions}

A schematic drawing of a formamide dimer is shown in Fig. 6. In Table 4 these bond lengths and angles are compared with data for related molecules.

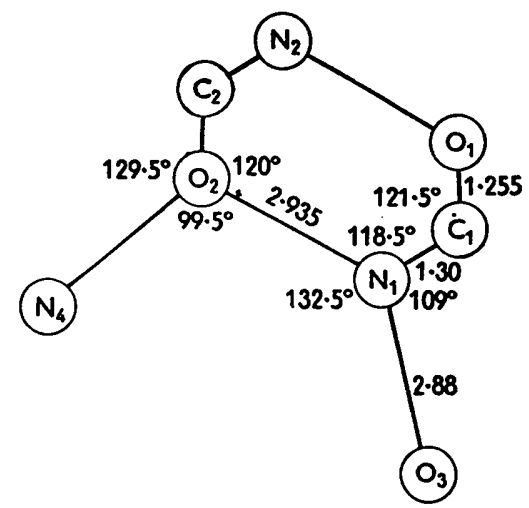

Fig. 6. Schematic drawing of formamide 'dimer'. Dimensions of bonds and bond angles are shown.

Table 4. Bond lengths and angles in formamide, and

\begin{tabular}{|c|c|c|c|c|}
\hline & $\begin{array}{l}\text { C-O } \\
(\AA)\end{array}$ & $\begin{array}{l}\mathbf{C}-\mathbf{N} \\
(\AA)\end{array}$ & $\begin{array}{c}\mathrm{O}-\mathrm{C}-\mathrm{N} \\
\left(^{\circ}\right)\end{array}$ & $\begin{array}{l}\text { Refer- } \\
\text { encet }\end{array}$ \\
\hline Formamide (monomer) & $1 \cdot 25_{5}$ & $1 \cdot 30_{0}$ & $121 \cdot 5$ & - \\
\hline Glutamine & $1 \cdot 27$ & $1 \cdot 28$ & 123 & (1) \\
\hline Acetamide & $1 \cdot 28$ & $1 \cdot 38$ & 122 & (2) \\
\hline Urea & $1 \cdot 26$ & $1 \cdot 34$ & 121 & (3) \\
\hline Glycyltyrosine. $\mathrm{HCl} \cdot \mathrm{H}_{2} \mathrm{O}$ & $1 \cdot 16$ & $1 \cdot 35$ & 132 & (4) \\
\hline$\beta$-Glycylglycine & $1 \cdot 23$ & $1 \cdot 29$ & 125 & (4) \\
\hline Cysteylglycine. $\frac{1}{2} \mathrm{NaI}$ & $1 \cdot 21$ & $1 \cdot 32$ & 107 & (4) \\
\hline Acetylglycin $\theta^{*}$ & $1 \cdot 24$ & $1 \cdot 32$ & 121 & (4) \\
\hline Diketopiperazine & $1 \cdot 25$ & $1 \cdot 33$ & - & (4) \\
\hline Cyanuric Acid* & $1 \cdot 21$ & $1 \cdot 35$ & 122 & (4) \\
\hline Oxamide & $1 \cdot 25$ & $1 \cdot 30$ & - & (5) \\
\hline Succinamide & $1 \cdot 22$ & $1 \cdot 40$ & 123 & (6) \\
\hline
\end{tabular}
comparison with other molecules

* Determined by three-dimensional Fourier synthesis.

$\dagger$ (1) Cochran \& Penfold, 1952; (2) Senti \& Harker, 1940; (3) Vaughan \& Donohue, 1952; (4) Smits \& Wiebenga, 1953; (5) Romers, 1953; (6) Pasternak, 1953. 


\section{Discussion of the structure}

In a large number of amides, imides and related compounds, the $\mathrm{C}-\mathrm{N}$ bonds appear to possess considerable double-bond character; their lengths are in the range $1 \cdot 28-1 \cdot 38 \AA$, i.e. much shorter than the $\mathrm{C}-\mathrm{N}$ singlebond length of 1.47 $\AA$ found in many amines (Donohue, 1952), see Table 4. The 1.30 $\AA \mathrm{C}-\mathrm{N}$ bond in formamide is near the lower limit observed for bonds of this type; evidently it possesses a high degree of double-bond character. This appears to be characteristic of the $\mathrm{C}-\mathrm{N}$ bonds in terminal amides; in glutamine, the $\mathrm{C}-\mathrm{N}$ bond length is $1.28 \AA$ (Cochran $\&$ Penfold, 1952) and in oxamide it is $1.30 \AA$ (Romers, 1953). The 1.38 $\AA \mathrm{C}-\mathrm{N}$ bond length reported for acetamide thus appears to be anomalously long and should probably be reinvestigated.

The three bonds emanating from the amide nitrogen atom have been found to be coplanar in a large number of peptide groups (Pauling, Corey \& Branson, 1951). They are also coplanar in oxamide (Romers, 1953). The three bonds from each nitrogen atom in formamide (i.e. $\mathrm{N}_{1}-\mathrm{C}_{1}, \mathrm{~N}_{1}-\mathrm{H} \cdots \mathrm{O}_{2}$, and $\mathrm{N}_{1}-\mathrm{H} \cdots \mathrm{O}_{3}$ ) are almost, but not quite, coplanar; i.e., in Fig. $6, \mathrm{O}_{3}$ is $0.2 \AA$ out of the plane formed by $\mathrm{C}_{1}, \mathrm{~N}_{1}$ and $\mathrm{O}_{2}$.

The hydrogen bonds which bind pairs of formamide molecules to form dimers are $2 \cdot 93_{5} \AA$ long; those between dimers are $2 \cdot \mathbf{8 8}_{\mathbf{0}} \AA$ long. The probable error of bond lengths is approximately $0.013 \AA$ and the difference between the two hydrogen bond lengths is therefore probably significant. Other $\mathrm{N}-\mathrm{H} \cdots \mathrm{O}$ bond lengths range from 2.68 to $3.14 \AA$ in length (Donohue, 1952); they are $2.86 \AA$ long in acetamide and range from 2.79 to $2.95 \AA$ in glutamine (Cochran \& Penfold, 1952). Hydrogen bond lengths of approximately 2.91 and $2.96 \AA$ have been computed from parameters reported for oxamide (Romers, 1953). 'Normal' $\mathrm{N}-\mathrm{H} \cdots \mathrm{O}$ bond lengths in compounds of this type appear to be close to $2.90 \AA$ in most cases, rather than $2.74 \AA$ as had been previously supposed (Pauling, Corey \& Branson, 1951). As can be seen in Fig. 6, the $\mathrm{C}-\mathrm{N}-\mathrm{O}$ bond angles are of two distinct types: $118.5^{\circ}$ and $109^{\circ}$.

The arrangement of molecules in crystalline formamide in puckered sheets of dimers may be compared with the three-dimensional polymeric arrangement reported for acetamide. On the other hand, both diamides which have thus far been investigated (oxamide and succinamide) appear to be arranged in the form of flat sheets. It would be interesting to investigate the arrangements of molecules in other mono and diamides to determine whether these arrangements are characteristic of crystals of these types.

The difference in length between the two types of hydrogen bonds in formamide may help explain the observed high dielectric constant of liquid formamide (Leader, 1951). Such a high dielectric constant would not be expected for liquid formamide if most of the molecules were associated in the form of dimers. It is reasonable to suppose that the longer of the two hydrogen bonds in the crystal is somewhat weaker and more easily broken on melting; if this is so then the predominance of chains of formamide molecules rather than sheets of dimers in the liquid is readily understood; under these circumstances the observed high dielectric constant of the liquid would be expected.

One other aspect of this structure is of interest. Cochran \& Penfold (1952) have noted that the C-N bond in glutamine appears to possess considerably more double-bond character than does the corresponding bond in acetamide. They have correlated this with the fact that hydrolysis of the $\mathrm{C}-\mathrm{N}$ group occurs more readily in glutamine than in acetamide; i.e. the increased double-bond character of the former renders it more susceptible to attack in reactions of this sort. Similarly, formamide hydrolyzes more readily than does acetamide (Hammet, 1940); this appears reasonable in terms of the observed higher doublebond character of $\mathrm{C}-\mathrm{N}$ bonds in formamide compared with acetamide.

The authors wish to thank Mr Kermit Hale of the Polytechnic Institute of Brooklyn for helpful suggestions concerning the design of the low-temperature Weissenberg camera. Also, we wish to thank the staff of the Watson Scientific Computing Laboratory for making I.B.M. facilities available to us. We particularly wish to express our gratitude to Prof. I. Fankuchen of the Polytechnic Institute of Brooklyn for many helpful suggestions in conjunction with this work. This effort was supported under contract N6onr-263 Task Order V, Project NR 017-604.

\section{References}

Bоотн, A. D. (1948). Fourier Technique in $X$-ray Organic Structure Analysis, p. 45. Cambridge: University Press. Cochran, W. \& Penfold, B. R. (1952). Acta Cryst. 5, 644.

Cruickshank, D. W. J. (1949). Acta Cryst. 2, 65.

Donohot, J. (1952). J. Phys. Chem. 56, 502.

HaMmet, L. P. (1940). Physical Organic Chemistry, p. 365. New York: McGraw Hill.

Leader, G. R. (195I). J. Amer. Chem. Soc. 73, 856.

Pasternak, R. A. (1953). Acta Cryst. 6, 808.

Pauling, L., Corey, R. B. \& Branson, H. R. (1951). Proc. Nat. Acad. Sci., Wash. 37, 206.

Post, B., Schwartz, R. S. \& Fankuchen, I. (1951). Rev. Sci. Instrum. 22, 218.

Romers, C. (1953). Acta Cryst. 6, 429.

Senti, F. \& Harker, D. (1940). J.Amer. Chem. Soc. 62, 2008.

Smits, D. W. \& Wiebenga, E. H. (1953). Acta Cryst. 6, 531.

Steinfink, H., Ladell, J., Post, B. \& Fankuchen, I. (1953). Rev. Sci. Instrum. 24, 882.

Vaughan, P. \& Donohue, J. (1952). Acta Cryst. 5, 530. 